\title{
ON THE IMBEDDABILITY OF CERTAIN COMPLEXES IN EUCLIDEAN SPACES
}

\author{
P. J. HILTON AND E. H. SPANIER ${ }^{1}$
}

1. Statement of results. Let $S^{p} \cup_{f} e^{q}(0<p<q)$ denote the complex obtained by attaching a $q$-cell $e^{q}$ to the $p$-sphere $S^{p}$ by means of a continuous map $f: S^{q-1} \rightarrow S^{p}$. Thus $S^{p} \cup_{f} e^{q}$ is the union of the mapping cylinder of $f$ with a cone over $S^{q-1}$. In this note we consider the problem of imbedding such a complex in euclidean space or, equivalently, in a sphere. It is clear that the mapping cylinder of $f$ can be imbedded in the join $S^{q-1} * S^{p}$, which is homeomorphic to $S^{p+q}$, and, therefore, $S^{p} \cup_{f} e^{q}$ can be imbedded in $S^{p+q+1}$. We shall give a condition which insures that this imbedding is into the lowest dimensional sphere in which it is possible to imbed $S^{p} \cup_{f} e^{q}$.

We say that the map $f: S^{q-1} \rightarrow S^{p}$ can be $S$-desuspended if there is a map $g: S^{q-2} \rightarrow S^{p-1}$ and an integer $k \geqq 0$ such that the $(k+1)$-fold suspension of $g$ is homotopic to the $k$-fold suspension of $f$ (denoted by $S^{k+1} g \simeq S^{k} f$ ). If there is no such $g$, then we say $f$ cannot be $S$ desuspended. Our main result is:

TheOREM I. Let $f: S^{q-1} \rightarrow S^{p}$ be a map which cannot be $S$-desuspended. Then $S^{p} \cup_{f} e^{q}$ can be imbedded in $S^{p+q+1}$ but not in $S^{p+q}$.

As an application of this theorem we see that if $f: S^{q-1} \rightarrow S^{2}(q \geqq 4)$ is such that no suspension of $f$ is null homotopic then $S^{2} \cup_{f} e^{q}$ can be imbedded in $S^{q+3}$ but not in $S^{q+2}$. In particular, the complex projective plane is homeomorphic to $S^{2} \cup_{f} e^{4}$ where $f: S^{3} \rightarrow S^{2}$ is a map of Hopf invariant one so it can be imbedded in $S^{7}$ but not in $S^{6}$.

In Theorem I note that if $p=q-1$ then any map $f: S^{p} \rightarrow S^{p}$ can be $S$-desuspended unless $p=1$ and $f$ has degree different from 0 and \pm 1 . If $p=1$ and $f$ has degree $\pm k$ where $k>1$, then $S^{1} \cup_{f} e^{2}$ has a finite nontrivial second cohomology group so if it could be imbedded in $S^{3}$ it would follow from the Alexander duality theorem that its complement in $S^{3}$ would have a finite nontrivial zero dimensional homology group, and this is impossible. Hence, Theorem I is proved in this case. Since any inessential map $f: S^{q-1} \rightarrow S^{p}$ can be $S$-desuspended, Theorem I does not apply for such maps. Therefore, to com-

Received by the editors September 8, 1959.

1 The authors were supported in part by Contract No. Nonr. 401 (20) and NSF Grant No. G-9103, respectively, during the period when this paper was in preparation. 
plete the proof of Theorem I we need only consider the range of values $1<p<q-1$.

The proof of Theorem I follows from an investigation of a weaker imbeddability which is better adapted to the $S$-category and duality therein. We say that a finite CW-complex $X$ can be $S$-imbedded in $S^{n}$ if there is a subcomplex $Y$ of $S^{n}$ and an integer $k \geqq 0$ such that $S^{k} X$ and $S^{k} Y$ have the same homotopy type. Let $i(X)$ denote the least integer $n$ such that $X$ can be $S$-imbedded in $S^{n}$. The function $i(X)$ has been considered in $[2 ; 3]$. Clearly $X$ cannot be imbedded in a sphere of dimension $i(X)-1$.

Given a map $f: S^{q-1} \rightarrow S^{p}(1<p<q-1)$ let $d(f)$ denote the greatest integer $k$ such that there is a map $g: S^{q-k-1} \rightarrow S^{p-k}$ with $S^{k+r} g \simeq S^{r} f$ for some $r \geqq 0$. Then $0 \leqq d(f) \leqq p$, and $d(f)=0$ if and only if $f$ cannot be $S$-desuspended while $d(f)=p$ if and only if some suspension of $f$ is null homotopic. In view of remarks made earlier Theorem I will follow if we can prove that if $f$ cannot be $S$-desuspended then

$$
i\left(S^{p} \cup_{f} e^{q}\right) \geqq p+q+1 .
$$

This is a consequence of the following:

TheOREM II. Let $1<p<q-1$. Then for any map $f: S^{q-1} \rightarrow S^{p}$ we have

$$
i\left(S^{p} \cup_{f} e^{q}\right)=p+q+1-d(f) .
$$

The proof of Theorem II uses the duality in the $S$-category [4] and some results of [1]. The case $q=p+3$ of this theorem has been established by F. P. Peterson and N. Stein (in a forthcoming paper) using secondary cohomology operations.

2. Proofs. Before we proceed to the proof of Theorem II we list some results which will be used in the proof. Throughout this section we shall assume $1<p<q-1$.

Lemma 1. If $S^{p} \cup_{f} e^{q}$ is of the same homotopy type as a suspension, then $f$ is homotopic to $\mathrm{Sg}$ for some map $\mathrm{g}: \mathrm{S}^{q-2} \rightarrow S^{p-1}$.

LEMMA 2. If $S^{p} \cup_{f} e^{q}$ and $S^{p} \cup_{0} e^{q}$ have the same homotopy type, then $f \simeq h g h^{\prime}$ where $h: S^{p} \rightarrow S^{p}$ and $h^{\prime}: S^{q-1} \rightarrow S^{q-1}$ are homotopy equivalences.

Lemma 1 is just Lemma 3.6 of [1], and Lemma 2 is proved in the proof of Lemma 3.6 of [1].

We let $X \# Y=X \times Y / X \vee Y$ denote the reduced product of $X$ and $Y$. A duality map

$$
v: S^{p} \# S^{r} \rightarrow S^{p+r}
$$


as defined in [4] reduces in the special case of spheres to just a homotopy equivalence between $S^{p} \# S^{r}$ and $S^{p+r}$. It is a consequence of Theorem (5.9) of [4] that if

$$
u: S^{r} \# S^{p} \rightarrow S^{p+r}, \quad v: S^{p} \# S^{r} \rightarrow S^{p+r}
$$

are duality maps there is an isomorphism

$$
D(u, v):\left\{S^{p}, S^{r}\right\} \approx\left\{S^{p}, S^{r}\right\}
$$

(where $\left\{S^{p}, S^{r}\right\}$ denotes the group of stable homotopy classes and is the direct limit of the groups $\pi_{p+k}\left(S^{r+k}\right)$ as $\left.k \rightarrow \infty\right)$. Furthermore, if $k$ is large enough and $f: S^{p+k} \rightarrow S^{r+k}, f^{\prime}: S^{p+k} \rightarrow S^{r+k}$ represent elements $\{f\},\left\{f^{\prime}\right\} \in\left\{S^{p}, S^{r}\right\}$, then $D(u, v)\{f\}=\left\{f^{\prime}\right\}$ if and only if the following diagram is homotopy commutative (see (5.11) of $[4]$ )

$$
\begin{array}{ccc}
S^{p+k} \# S^{p+k} & 1 \# f & \\
f^{\prime} \# 1 \downarrow & S^{p+k} \# S^{r+k} \\
S^{r+k} \# S^{p+k} & \stackrel{u_{k, k}}{\longrightarrow} S^{p+r+2 k}
\end{array} .
$$

The following answers a question raised on page 270 of [3].

Lemma 3. The map $D(u, v):\left\{S^{p}, S^{r}\right\} \approx\left\{S^{p}, S^{r}\right\}$ is equal to \pm 1 (where 1 denotes the identity map of $\left\{S^{p}, S^{r}\right\}$ ).

Proof. Since $v_{k, k}$ and $u_{k, k}$ are homotopy equivalences and the map $T: S^{p+k} \# S^{r+k} \rightarrow S^{r+k} \# S^{p+k}$ which interchanges the two factors is also a homotopy equivalence, it follows that $v_{k, k} \simeq h u_{k, k} T$ where $h: S^{p+r+2 k} \rightarrow S^{p+r+2 k}$ is a homotopy equivalence (so has degree \pm 1 ). From the homotopy commutativity of the diagram above we see that

$$
u_{k, k}\left(f^{\prime} \# 1\right) \simeq h u_{k, k} T(1 \# f) \simeq u_{k, k} h^{\prime} T(1 \# f)
$$

where $h^{\prime}$ is a homotopy equivalence of $S^{r+k} \# S^{p+k}$ with itself. Since $u_{k, k}$ is a homotopy equivalence, this implies that

$$
f^{\prime} \# 1 \simeq h^{\prime} T(1 \# f) \text {. }
$$

Let $T^{\prime}$ be the map of $S^{p+k} \# S^{p+k}$ into itself which interchanges the two factors. Then $T(1 \# f)=(f \# 1) T^{\prime}$ so

$$
f^{\prime} \# 1 \simeq h^{\prime}(f \# 1) T^{\prime} .
$$

Since $f^{\prime} \# 1=S^{p+k} f^{\prime}, f \# 1=S^{p+k} f$ and $h^{\prime}, T^{\prime}$ are homotopy equivalences, the above equation implies that $\left\{f^{\prime}\right\}= \pm\{f\}$ and completes the proof.

Proof of Theorem II. Let $g: S^{q-k-1} \rightarrow S^{p-k}$ be a map which cannot be $S$-desuspended and such that $S^{k+r} g \simeq S^{r} f$ (so $d(f)=k$ ). Then as we 
saw in the first paragraph of $\S 1, S^{p-k} \cup_{\theta} e^{q-k}$ can be imbedded in $S^{p+q-2 k+1}$ so $S^{k}\left(S^{p-k} \cup_{g} e^{q-k}\right)$ can be imbedded in $S^{p+q-k+1}$. Since $S^{r}\left(S^{k} g\right) \simeq S^{r} f$ it follows that $S^{r}\left(S^{k}\left(S^{p-k} \cup_{g} e^{q-k}\right)\right)$ has the same homotopy type as $S^{r}\left(S^{p} \cup_{f} e^{q}\right)$ so $S^{p} \cup_{f} e^{q}$ can be $S$-imbedded in $S^{p+q-k+1}$ showing that $i\left(S^{p} \cup_{f} e^{q}\right) \leqq p+q+1-d(f)$.

To prove the opposite inequality note that if $d(f)=p$ there is no subset $Y$ of $S^{q}$ such that $S^{k} Y$ and $S^{k}\left(S^{p} \cup_{f} e^{q}\right)$ have the same homotopy type because such a $Y$ would have to have $H^{p}(Y)$ and $H^{q}(Y)$ different from 0 , and there is no subset of $S^{q}$ like that. Therefore, if $d(f)=p$, $i\left(S^{p} \cup_{f} e^{q}\right)=q+1$.

Therefore, we may assume $d(f)<p$. Since $2 \leqq p<q-1$ we must also have $d(f)<p-1$. Assume $i\left(S^{p} \cup_{f} e^{q}\right)<p+q+1-d(f)$ so $S^{p} \cup_{f} e^{q}$ can be $S$-imbedded in $S^{p+q-d(f)}$. Let $X$ be a subcomplex of $S^{p+q-d(f)}$ such that $S^{r} X$ has the same homotopy type as $S^{r}\left(S^{p} \cup_{f} e^{q}\right)$. Let $X^{\prime}$ be a deformation retract of $S^{p+q-d(f)}-X$. Then $X^{\prime}$ is a $(p+q-d(f)-1)$ dual ${ }^{2}$ of $X\left(5.1\right.$ of [4]) so $X^{\prime}$ is a $(p+q+r-d(f)-1)$-dual of $S^{r} X$ and, hence, also of $S^{r}\left(S^{p} \cup_{f} e^{q}\right)$. Let $u: S^{p} \# S^{q-1} \rightarrow S^{p+q-1}, v: S^{q-1} \# S^{p}$ $\rightarrow S^{p+q-1}$ be duality maps. Then $D(u, v)\{f\}=\{g\}$ where $\{g\}= \pm\{f\}$ by Lemma 3. By (6.10) of [4] $S^{p} \cup_{f} e^{q}$ and $S^{p} \cup_{g} e^{q}$ are $(p+q)$-dual. Then $S^{r}\left(S^{p} \cup_{f} e^{q}\right)$ and $S^{p} \cup_{g} e^{q}$ are $(p+q+r)$-dual. Since $S^{d(f)+1} X^{\prime}$ is also $(p+q+r)$-dual to $S^{r}\left(S^{p} \cup_{f} e^{q}\right)$, it follows that, for some $k$, we have $S^{k+d(f)+1} X^{\prime}$ is of the same homotopy type as $S^{k}\left(S^{p} \cup_{g} e^{q}\right)$. Now $S^{1} X^{\prime}$ is of the same homotopy type as $S^{p-d(f)} \cup_{g_{1}} e^{q-d(f)}$ for some $g_{1}: S^{q-d(f)-1}$ $\rightarrow S^{p-d(f)}$ (because it is simply connected and has nontrivial integral homology groups only in dimensions $p-d(f)$ and $q-d(f)$, and these are infinite cyclic). By Lemma $1, g_{1} \simeq S g_{1}^{\prime}$ for some $g_{1}^{\prime}: S^{q-d(f)-2}$ $\rightarrow S^{p-d(f)-1}$, and by Lemma $2, S^{k} g \simeq \pm S^{k+d(f)} g_{1}$ so $S^{k} g \simeq \pm S^{k+d(f)+1} g_{1}^{\prime}$. Therefore, $d(g) \geqq d(f)+1$. Since $\{g\}= \pm\{f\}, d(g)=d(f)$, and we have arrived at a contradiction.

\section{REFERENCES}

1. I. Bernstein and P. J. Hilton, Category and generalized Hopf invariants, Illinois J. Math. (to appear.)

2. F. P. Petersen, Some non-imbedding problems, Bol. Soc. Mat. Mexicana, (II) vol. 2 (1957) pp. 9-15.

3. E. Spanier, Duality and the suspension category, Symposium Internacional de Topologia Algebraica, Mexico City, 1958, pp. 259-272. 378.

4. - Function spaces and duality, Ann. of Math. (2) vol. 70 (1959) pp. 338-

Cornell University and University of Birmingham, UNiversity of California, Berkeley

${ }^{2}$ This is the notational usage of [4]. In terms of the definition and terminology of [3] $X^{\prime}$ is a $(p+q-d(f))-$ dual of $X$. 